\title{
Intraventricular haemorrhage and periventricular leucomalacia: ultrasound and autopsy correlation
}

\author{
J Q TROUNCE, D FAGAN, AND M I LEVENE
}

Department of Child Health, Leicester University Medical School, and Department of Histopathology, Queen's Medical Centre, Nottingham

SUMMARY The brains of 30 infants who died after at least one real time ultrasound scan were examined after fixation. The ultrasound diagnosis of either periventricular haemorrhage or periventricular leucomalacia was compared with the macroscopic and histological appearances. Each hemisphere was considered separately for both periventricular haemorrhage and periventricular leucomalacia. The accuracy of ultrasound diagnosis for periventricular haemorrhage was $88 \%$, with sensitivity of $91 \%$ and specificity of $85 \%$. The accuracy for periventricular leucomalacia was $90 \%$, with sensitivity of $85 \%$ and specificity of $93 \%$. Ultrasound was shown to diagnose the entire range of periventricular leucomalacia lesions. Three hemispheres showed the appearance of prolonged flare, and this correlated with extensive spongiosis and microcalcification of the periventricular white matter, although no macroscopic lesion was seen.

There is now clear evidence that the long term neurodevelopmental prognosis for neonates with intraventricular haemorrhage is significantly better than for those with periventricular leucomalacia (PVL). ${ }^{1-3}$ If ultrasound imaging is to be effective in predicting prognosis then clearly an accurate differentiation of the pathological entities must be possible on scanning. Previous workers have reported accurate correlation between ultrasound demonstration of intraventricular haemorrhage and its finding on computed tomography or at autopsy, ${ }^{4-9}$ but in none of these studies was an attempt made to differentiate parenchymal lesions due to 'extension of haemorrhage' from PVL. As part of a prospective study of intraventricular haemorrhage and PVL in the preterm neonate we have had the opportunity to correlate ultrasound scan and autopsy findings and we now report our results.

\section{Patients and methods}

Over a 16 month period (1 January 1984 to 30 April 1985) 200 neonates of birth weight $\leqslant 1500 \mathrm{~g}$ admitted to two neonatal intensive care units (Leicester Royal Infirmary and Nottingham City Hospital) had regular cranial ultrasound scans. At least one scan had to have been performed during life for the patient to be included in the study. In both centres an ATL Mark III real time sector scanner fitted with a $3 \cdot 5,5$, and
7.5 $\mathrm{MHz}$ multifrequency scanhead was used. Ultrasound diagnoses based on our previous definitions ${ }^{10}$ were made before autopsy in all cases. The brain was removed complete at the time of postmortem examination and suspended in $10 \%$ formalin for at least one month before sectioning. Coronal cuts were made at $1 \mathrm{~cm}$ intervals and the macroscopic appearances noted. Histological examination of the periventricular area was performed in most cases. For the purposes of analysis, each hemisphere was considered separately. The number of true positive (TP), true negative (TN), false positive (FP), and false negative (FN) ultrasound diagnoses for each cerebral hemisphere was tabulated. The accuracy of ultrasound was measured by calculating TP+TN/ total number of hemispheres examined. Sensitivity was calculated as $\mathrm{TP} /(\mathrm{TP}+\mathrm{FN})$ and specificity as $\mathrm{TN} /(\mathrm{FP}+\mathrm{TN})$.

To show the range of PVL lesions we have included five additional babies who were not included in our previous study. ${ }^{10}$ Two of these had scans performed soon after death but had no in vivo scan, and a further two preterm infants had birth weights that had excluded them from the previous study. The final infant was born at 29 weeks' gestation and suffered severe arrhythmias in the first $\mathbf{2 4}$ hours of life related to hyperkalaemia. An ultrasound scan at the age of 17 days showed extensive bilateral cystic PVL, involving temporal 
and occipital regions, and this was still present when she was last scanned at the age of 7 weeks. She died suddenly aged 6 months and thus illustrates the more long term evolution of PVL.

\section{Results}

Forty two of the 200 study babies died, and all deaths occurred before discharge from hospital. Postmortem examination was performed in 31 of the 43 deaths, but in one infant the brain was too soft to fix. The results of the correlation between autopsy and ultrasound examinations are shown in Tables 1 and 2 for the 30 infants with complete postmortem data. Histological examination was performed on 25 brains.

Haemorrhage. Haemorrhage into the germinal matrix or ventricle or extension into the cerebral parenchyma was diagnosed both by ultrasound and subsequently at autopsy in 31 hemispheres. The results are summarised in Table 1 . A false positive ultrasound diagnosis of haemorrhage was made in four hemispheres (three infants) in which postmortem examination subsequently showed a normal hemisphere. In three the ultrasound appearance was thought to consist of haemorrhage in the region of the germinal matrix, and the other seemed to show the appearances of more extensive clot in the lateral ventricles. A false negative diagnosis of haemorrhage was made in three hemispheres (two babies) (Table 2). All three hemispheres contained a small

Table 1 Correlation between ultrasound and postmortem diagnoses for the study group of 60 hemispheres. As each hemisphere may have more than one form of disease the numbers do not add up to 60. Values are No (\%)

\begin{tabular}{lccc}
\hline Ultrasound observations & \multicolumn{3}{l}{ Postmortem observations } \\
\cline { 2 - 4 } & Normal & PVH & $P V L$ \\
\hline Normal & $22(78)$ & $3(11)$ & $3(11)$ \\
PVH & $4(11)$ & $31(89)$ & 0 \\
PVL & $3(15)$ & 0 & $17(85)$ \\
\hline
\end{tabular}

$\mathrm{PVH}=$ Periventricular haemorrhage; $\mathrm{PVL}=$ periventricular leucomalacia .

Table 2 Correlation between postmortem and ultrasound diagnoses for the 60 hemispheres. Values are No (\%)

\begin{tabular}{llrc}
\hline Postmortem observations & \multicolumn{4}{l}{ Ultrasound observations } \\
\cline { 2 - 4 } & Normal & PVH & PVL \\
\hline Normal & $22(76)$ & $5(17)$ & $2(7)$ \\
PVH & $3(9)$ & $31(91)$ & 0 \\
PVL & $3(15)$ & 0 & $17(85)$ \\
\hline
\end{tabular}

PVH=Periventricular haemorrhage; $\mathrm{PVL}=$ periventricular leucomalacia. germinal matrix haemorrhage that had ruptured into the ventricles. The accuracy of ultrasound diagnosis of haemorrhage was $88 \%$. The sensitivity of diagnosis was $91 \%$ and specificity $85 \%$.

Ischaemic lesions. Echodensity in the periventricular white matter detected by ultrasound and thought not to be parenchymal extension of periventricular haemorrhage was seen in 20 hemispheres, and PVL lesions were diagnosed at autopsy in 20 hemispheres (Tables 1 and 2). The accuracy of the ultrasound diagnosis was $90 \%$. A false positive ultrasound diagnosis of abnormal periventricular echodensity was made in three hemispheres (two infants). One child was only scanned once and the other had three scans, and mild bilateral flare was thought to be present on each occasion. A false negative ultrasound diagnosis of PVL was made in three hemispheres (two infants). In two hemispheres there was non-haemorrhagic PVL and in one hemisphere extensive haemorrhagic PVL was found and not previously detected by ultrasound. The sensitivity of ultrasound in the diagnosis of PVL was $85 \%$ and specificity $93 \%$.

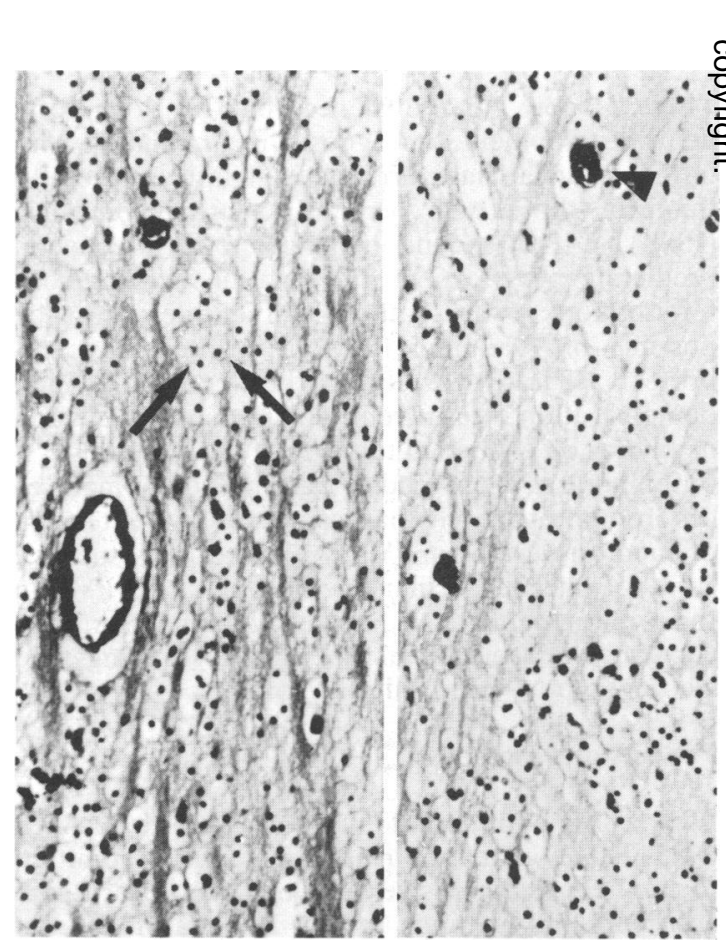

Fig. 1 Spongiosis and microcalcification in an infant with prolonged flare. Haematoxylin and eosin stain, showing generalised spongiosis of periventricular white matter (arrow) and microcalcification (arrow head). 

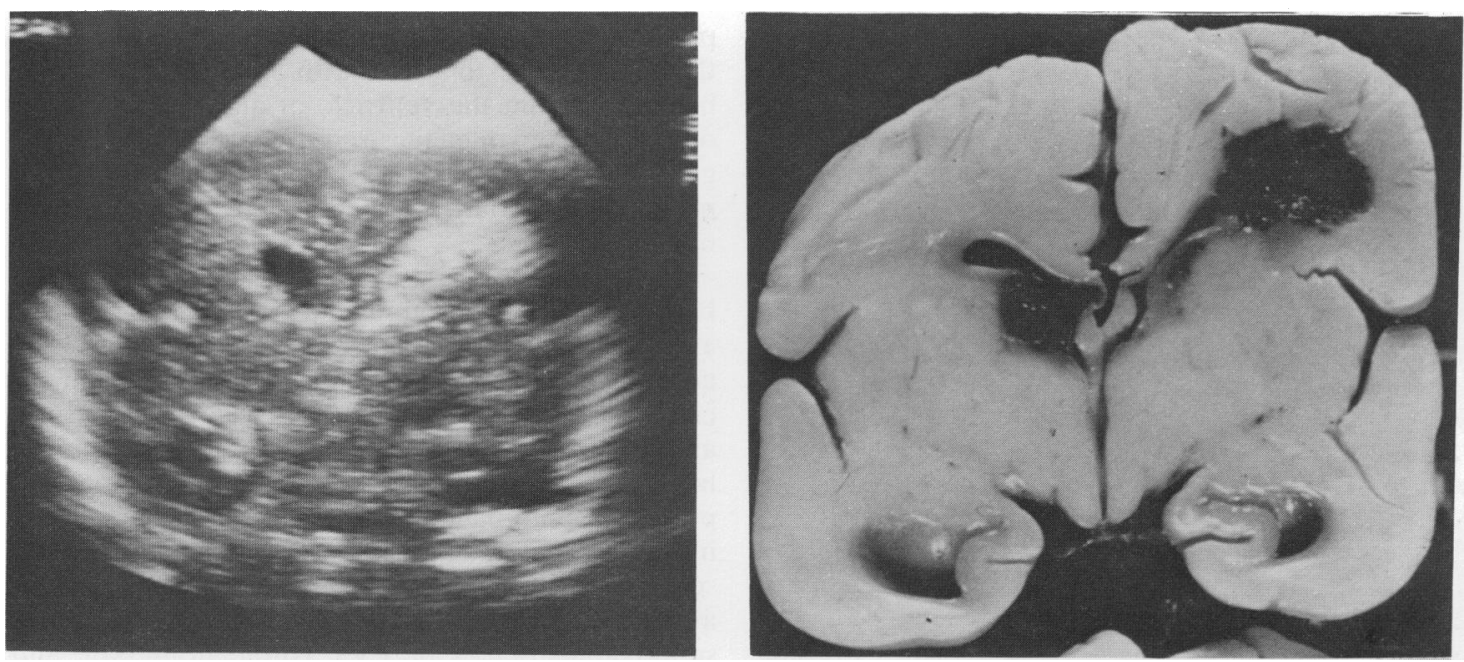

Fig. 2 Precystic periventricular leucomalacia. Coronal ultrasound view (left) showing triangular shaped echodensity in the right hemisphere. The pathological correlate (right) confirms haemorrhagic infarction in the region of the echodensity. There is a small unruptured subependymal haemorrhage on the right side and a somewhat larger lesion involving the left subependymal region.

Range of PVL lesions. To show the variety of PVL lesions an additional five infants have been considered together with the study infants. The ultrasound appearances we have described as prolonged flare $^{10}$ occurred in two infants (three hemispheres) whose brains were examined at autopsy. The flares

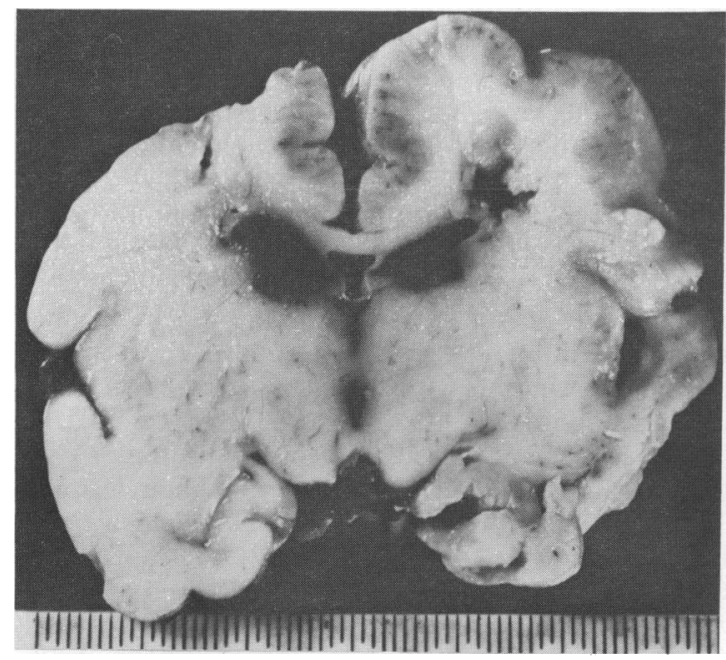

Fig. 3 Cystic periventricular leucomalacia, showing a single cavity in the right periventricular region separate from the lateral ventricle. Intraventricular clot is also present arising from bilateral subependymal haemorrhages. persisted for two weeks and three months, respectively, and were present on the last scan before death. No macroscopic abnormality was seen in either brain, but on histological examination extensive spongiosis and microcalcification was present in the periventricular white matter corresponding to the areas of echodensity (Fig. 1).

At autopsy one infant showed the characteristic 'white spots' described by Banker and Larroche. ${ }^{11}$ On ultrasound examination persistent bilateral flare was noted and it was thought that small echofree cavitations subsequently occurred shortly before death. The cysts were not confirmed at autopsy, but the echofree areas may have corresponded to the macroscopic white spots of lipid accumulation.

Seven infants (eight hemispheres) showed the pathological appearance of haemorrhagic infarction at autopsy. These infants died between the ages of 5 hours and 13 days. In all cases the floor of the ventricle appeared intact. In six of these infants in whom an ultrasound diagnosis of precystic PVL was made the necropsy appearance was consistent with haemorrhagic infarction in the region of the echodensity (Fig. 2).

Three infants were found to have periventricular cysts at autopsy. In all cases the initial ultrasound appearance was of a triangular shaped echodensity with its apex towards the angle of the lateral ventricle, from which echofree cavities developed in two of the three cases. A unilateral periventricular cyst was not detected in one baby on scanning, 


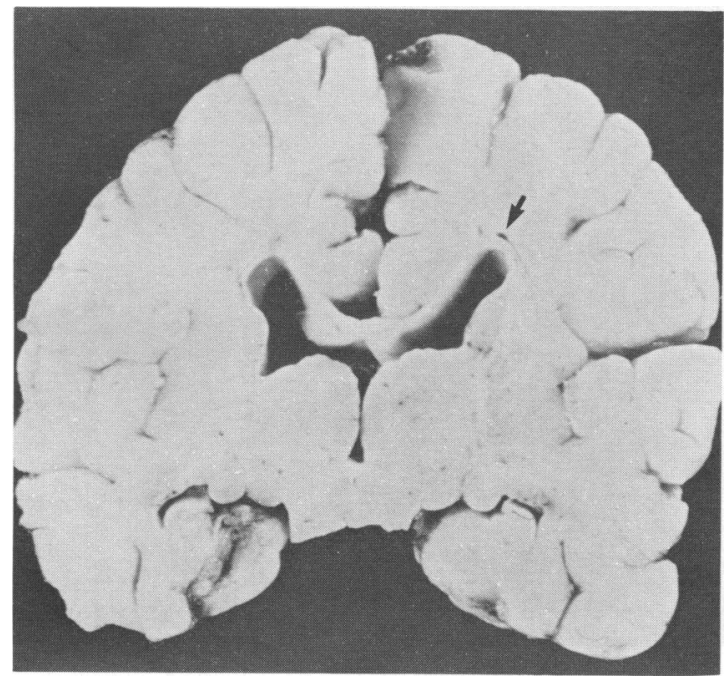

Fig. 4 Resolving cavities. Coronal section from an infant shown to have extensive cystic periventricular leucomalacia in the newborn period. Autopsy shows a collapsed cavity in the right periventricular region (arrowed). There is also compensatory ventricular enlargement.

although a flare had been seen in the corresponding area. This infant was scanned only once during life (Fig. 3).

One infant died aged 6 months of sudden infant death syndrome who at discharge from the neonatal unit showed extensive echofree cavitation in the periventricular white matter of both hemispheres. At autopsy there was irregular, ventricular dilatation, but the posteriorly placed cysts remained only as flattened cavities (Fig. 4), while the anterior cysts could no longer be seen at all.

\section{Discussion}

We have shown that real time ultrasound is an extremely good method for diagnosing both intraventricular haemorrhage (accuracy $88 \%$ ) and PVL (accuracy $90 \%$ ) in a series of 30 brains. Previous studies have produced similar autopsy correlation with the ultrasound diagnosis of periventricular haemorrhage in the region of $90 \%$ accuracy. ${ }^{5-9}$ In the two infants we report here with a false negative diagnosis of haemorrhage there was an interval of a few hours between the last scan and death, and the bleeding may have occurred during this time. A false positive diagnosis of haemorrhage was made in four hemispheres (three babies), all of whom died within 24 hours of birth. One baby had a massive contralateral parenchymal haemorrhage, and it is possible that on scanning we detected spread of thrombus from this site with no actual focus of bleeding within the ventricle in question. Szymonowicz and her colleagues reported congestion of the germinal matrix region that simulated germinal matrix haemorrhage, ${ }^{9}$ but this was not present in our infants.

Less information is available on the correlation between the ultrasound diagnosis of PVL and autopsy data. Nwaesei and colleagues reported $78 \%$ accuracy between ultrasound demonstration of increased echos or cysts in the periventricular region and the finding of PVL at autopsy in the corresponding site. ${ }^{12}$ In addition, they made the important point that both haemorrhagic and non-haemorrhagic infarction may produce identical changes on ultrasound. More recently, Fawer et al reported excellent autopsy correlation in five infants with the ultrasound appearances of PVL (three non-cystic and two cystic), and there were no false negative diagnoses. ${ }^{13}$ In our study ultrasound failed to detect PVL in two babies (three hemispheres). In one hemisphere the changes were early and in the other two there were histological abnormalities only (spongiosis and reactive astrocytosis but no microcalcification). A discrete cyst was missed in oneo infant whose only scan was performed 48 hour\$ before his death aged 5 days. The scan showed some flaring in the area where the cyst was subsequently observed at postmortem examination. The cyst undoubtedly would have been present at the time of the scan, and we cannot explain why it was not detected, but distortion may possibly have occurred during fixation to give a cystic appearance larger than it may have been in vivo.

Armstrong and Norman reported bleeding into the ischaemic area in $25 \%$ of their infants with PVL, ${ }^{14}$ and we found this in six of $34(18 \%)$ hemispheres. There is, however, considerable controversy concerning the differentiation between parenchymal extension of intraventricular haemorrhage and bleeding into a previously ischaemic lesion. Rushton and colleagues have recently reported eight infants with parenchymal abnormalities on ultrasound and at autopsy all showed PVL. ${ }^{15}$ These authors proposed that this is the underlying aetiology to all parenchymal haemorrhagic lesions. The evidence for this is still far from conclusive and it is beyond the scope of this study to resolve this question.

We have attempted to differentiate parenchymal extension of intraventricular haemorrhage from PVL by ultrasound scanning and report the relative incidence in a separate paper. ${ }^{10}$ The autopsy data reported here confirm that all cases of haemorrhagic infarction diagnosed by ultrasound were subse- 
quently found to be correct. No infant with parenchymal extension of intraventricular haemorrhage died in the acute stages and correlation in this group was not possible. We are encouraged, however, by the accuracy of ultrasound in diagnosing the range of PVL lesions reported here. This supports the validity of the ultrasound definitions that we have previously reported and used to evaluate prospectively a large group of very low birthweight infants. ${ }^{10}$

We have had the opportunity of observing a variety of appearances in the immature brain that represent the range of perinatal ischaemic injury. The histologic appearances of PVL have been described originally by Virchow in $1867^{16}$ and later by Banker and Larroche. ${ }^{11}$ These appearances may be present without macroscopic abnormality, and we have shown that ultrasound can show both gliosis and the 'white spots' of Banker and Larroche. Haemorrhagic infarction and subsequent cavitation is also readily diagnosed by ultrasound imaging. Two cases have been reported of infants showing extensive cavitation on sequential ultrasound scans in whom the echofree cystic lesions disappeared by 6 months. ${ }^{1718}$ We report here pathological correlation with this sequence of scan findings. With collapse of the cystic cavities, compensatory ventricular dilatation occurred due to cerebral atrophy. As far as we know this is the only such pathological correlation reported to date.

In conclusion, real time ultrasound scanning seems to be an excellent method for identifying the entire range of PVL lesions. It is of particular interest that apparently normal macroscopic brain appearances are echodense in the presence of microscopic calcification and gliosis. In addition, both the specificity and sensitivity of ultrasound is high for the diagnosis and exclusion of these lesions. We have confirmed that the ultrasound distinction between types of PVL as described elsewhere ${ }^{10}$ is a realistic and reliable proposition.

We are grateful to the staff of the LRI and NCH neonatal units for their cooperation and to Dr Pippa Stocks for the collection of specimens. We thank Professor J S Wigglesworth for his opinion on the brain of the cot death infant.

Dr Trounce is funded by the Spastics Society.

\section{References}

' Levene MI, Dubowitz LMS, de Crespigny LCh. Classifying intraventricular haemorrhage. Lancet 1983; ii:49.
2 De Vries LS. Dubowitz LMS. Dubowitz V, et al. Predictive value of cranial ultrasound in the newborn baby: a reappraisal. Lancet 1985:ii:137-40.

${ }^{3}$ Weindling AM, Rochefort MJ, Calvert SA, Fok T-F, Wilkinson A. Development of cerebral palsy after ultrasonographic detection of periventricular cysts in the newborn. Dev Med Child Neurol 1985;27:800-6.

${ }^{4}$ Mack LA. Wright K. Hirsch JH, et al. Intracranial hemorrhage in premature infants: accuracy of sonographic evaluation. $A J R$ 1981:137:245-50.

5 Babcock DS, Bove KE, Han BK. Intracranial hemorrhage in premature infants: sonographic-pathologic correlation. AJNR 1982;3:309-17

- Levene MI, Fawer C-L, Lamont R. Risk factors in the development of intraventricular haemorrhage in the preterm neonate. Arch Dis Child 1982;57:410-7.

7 Thorburn RJ, Lipscomb AP, Reynolds EOR, et al. Accuracy of imaging of the brains of newborn infants by linear array realtime ultrasound. Early Hum Dev 1982:6:31-46.

${ }^{8}$ Pape KE, Bennett-Britton S, Szymonowicz W, Martin DJ, Fitz CR, Becker L. Diagnostic accuracy of neonatal brain imaging: a postmortem correlation of computed tomography and ultrasound scans. J Pediatr 1983:102:275-80.

" Szymonowicz W, Schafler K. Cussen LJ, Yu VYH. Ultrasound and necropsy study of periventricular haemorrhage in preterm infants. Arch Dis Child 1984;59:637-42.

10 Trounce JQ, Rutter N, Levene MI. Periventricular leucomalacia and intraventricular haemorrhage in the preterm neonate. Arch Dis Child 1986:61:1196-202.

"Banker BQ. Larroche J-C. Periventricular leukomalacia of infancy. A form of neonatal anoxic encephalopathy. Arch Neurol 1962;7:386-410.

12 Nwaesei CG, Pape KE, Martin DJ, Becker LE, Fitz CR. Periventricular infarction diagnosed by ultrasound: a postmortem correlation. J Pediatr 1984:105:106-10.

13 Fawer C-L, Calame A, Perentes E, Anderegg A. Periventricular leukomalacia: a correlation study between real time ultrasound and autopsy findings. Neuroradiology 1985:27:292-300.

it Armstrong D, Norman MG. Periventricular leucomalacia in neonates. Complications and sequelae. Arch Dis Child 1974:49: $367-75$.

15 Rushton DI, Preston PR, Durbin GM. Structure and evolution of echo dense lesions in the neonatal brain. Arch Dis Child 1985;60:798-808.

16 Virchow R. Zur pathologischen Anatomie des Gehirns. 1. Congenitale Encephalitis und Myclitis. Virchows Archiv. A: Pathology. Pathologische Anatomie 1867:38:129-42.

17 Bowerman RA. Donn SM. DiPietro MA, D'Amato CJ, Hicks SP. Periventricular leukomalacia in the pre-term newborn infant: sonographic and clinical features. Radiology 1984;151: 383-8.

18 Dubowitz LMS, Bydder GM, Mushin J. Developmental sequence of periventricular leukomalacia. Arch Dis Child 1985;60: $349-55$.

Correspondence to Dr M I Levene, Neonatal Unit. Leicester Royal Infirmary, Leicester LE2 7LX, England.

Received 6 August 1986 